\title{
Referência e Contrarreferência na Atenção Secundária em Odontologia em Campinas, SP, Brasil
}

\author{
Referencing and counter-referencing in specialized dental health \\ procedures in Campinas in the state of São Paulo, Brazil
}

Fabiana de Lima Vazquez ${ }^{1}$

Luciane Miranda Guerra ${ }^{1}$

Eduardo de Sant'Anna Vítor ${ }^{1}$

Glaucia Maria Bovi Ambrosano ${ }^{1}$

Fábio Luís Mialhe ${ }^{1}$

Marcelo de Castro Meneghim ${ }^{1}$

Antonio Carlos Pereira ${ }^{1}$

\footnotetext{
${ }^{1}$ Faculdade de Odontologia de Piracicaba. Unicamp. Av. Limeira 901, Piracicaba. 13.414-903 Piracicaba SP Brasil. apereira@fop.unicamp.br
}

\begin{abstract}
This article aims to evaluate the referencing of the demand for specialized care in oral health in Campinas in relation to the distribution of treatment and the influence of socio-economic variables in addition to the perception and information available to users in relation to counter-referencing. It is an observational exploratory study, selecting two Health Administrative Regions: one with a Center for Dental Specialties and one without, divided into two phases: 1) all referrals to secondary care were collected from all Oral Health teams as well as population and socio-economic data and distribution of referrals; 2) interview with 331 users, checking the counterreferencing, quality of service and reasons for $a b$ sences or withdrawals. Regarding the counterreferencing, the majority of users who received the specialized treatments were well attended, and waited less than one month for appointment scheduling. The management model did not statistically influence access to secondary care in oral health. It was found that in a given Health Administrative Region the discrepancies were less evident and there was a correlation coefficient of demand attended with socio-economic variables, in addition to a lower percentage of non-attendance, demonstrating a greater commitment to integrity and fairness.
\end{abstract}

Key words Oral health, Access to health services, Equity
Resumo O artigo tem por objetivo avaliar o referenciamento da demanda de atenção especializada em saúde bucal em Campinas em relação à distribuição da oferta e influência de variáveis socioeconômicas, além de percepção e informações dos usuários sobre à contrarreferência. Estudo observacional exploratório, selecionando duas Regiões Administrativas de Saúde (RAS): uma com Centro de Especialidades Odontológicas (CEO), e outra sem CEO, dividido em 2 fases: 1 ) todos os encaminhamentos para atenção secundária foram coletados de todas as equipes de Saúde Bucal, além de dados populacionais, socioeconômicos e distribuição dos encaminhamentos; 2) entrevista com 331 usuários, verificando-se a contrarreferência, qualidade de atendimento e razões para faltas ou desistências. Em relação à contrarreferência, a maioria dos usuários realizou os tratamentos especializados, os quais qualificaram como bom e esperaram menos de 1 mês para o agendamento. $O$ modelo de gestão não influenciou estatisticamente no acesso à atenção secundária em saúde bucal. As discrepâncias foram menos evidentes em uma RAS, havendo correlação dos coeficientes de tratamento e variáveis socioeconômicas, além de menor percentual de faltas, demonstrando maior compromisso com a integralidade e equidade.

Palavras-chave Saúde bucal, Acesso aos serviços de saúde, Equidade 


\section{Introdução}

Nas últimas duas décadas, importantes conquistas foram alcançadas pela saúde bucal no Brasil. Além da queda significativa na prevalência de cárie aos 05 e aos 12 anos de idade, ocorrida entre 2003 e 2010, verificou-se também uma inversão de tendência, com as extrações de dentes cedendo espaço aos tratamentos restauradores ${ }^{1}$. Tais avanços foram relevantes, principalmente considerando a desassistência em saúde bucal, originada no Brasil por modelos de atenção fragmentados e focalizados adotados até o final do século $\mathrm{XX}^{2,3}$.

A progressiva implantação da Estratégia de Saúde da Família (ESF) no Brasil, verificada entre 1998 e 2011, refletiu-se na ampliação do direito de acesso e caracterizou importante passo na inversão do modelo de atenção em saúde bucal ${ }^{4,5}$.

A implementação da atenção secundária atualmente é um grande desafio para o alcance da integralidade no Sistema Único de Saúde (SUS), sendo necessário o desenvolvimento do fluxo de referência e contrarreferência, através do qual as informações e os usuários trafegam no sistema. As dificuldades encontradas nesse trajeto são definitivamente os entraves da integralidade. Superá-las e/ou amenizá-las, pode encurtar o caminho para a resolução das necessidades em saúde bucal.

Assim, os municípios estabeleceram diversas formas de implantação do nível secundário de acordo com a viabilidade. Entretanto, a principal estratégia adotada para a atenção especializada em saúde bucal foi a implantação dos Centros de Especialidades Odontológicas (CEO). Uma de suas diretrizes foi a ampliação e a qualificação da atenção secundária e terciária em saúde bucal, fundamentada no fato de que, no âmbito do SUS, os serviços odontológicos especializados não ultrapassaram, até o ano de 2003, o percentual de $3,5 \%$ do total de procedimentos clínicos, evidenciando que a expansão da atenção secundária e terciária não acompanhou o crescimento da oferta de serviços odontológicos básicos ${ }^{6,7}$.

Mas na inversão do modelo de atenção, o processo de trabalho necessita estruturar-se coletivamente e não mais de forma hegemônica como antes ${ }^{8}$. $\mathrm{O}$ foco da produção não poderá ser mais a doença, e sim o indivíduo.

Para o alcance da integralidade, é necessária a organização do processo de trabalho dentro desses modelos. A estruturação do sistema de referência e contrarreferência - ainda num estágio de pouco desenvolvimento tanto em relação aos seus possíveis sentidos teóricos, quanto no que refere à efetivação e divulgação de experiências exitosas ou não tem importância crucial nesse processo $^{9}$

A partir de meados da década de 80 há relatos de problemas na organização do sistema de referência e contrarreferência, tais como a falta de informação da população, problemas organizacionais e problemas de integração e comunicação entre os vários níveis de atenção ${ }^{10}$. Isso parece ser particularmente importante quando se verifica que muitos municípios não registram informações sobre os respectivos sistemas de referência e contrarreferência nos Planos Municipais de Saúde e nem no Datasus ${ }^{11}$.

Assim, a busca por informações acerca das formas de organização da demanda na atenção secundária, modelos de gestão, acessibilidade e percepção do usuário são importantes para o planejamento em saúde.

Dessa forma os objetivos do presente estudo foram: avaliar o sistema de referência e contrarreferência e o processo de trabalho na atenção secundária em saúde bucal em duas Regiões Administrativas de Saúde (RAS) no município de Campinas.

\section{Metodologia}

Este projeto foi aprovado pelo Comitê de Ética em Pesquisa da Faculdade de Odontologia de Piracicaba e pelo Comitê de Ética da Secretaria Municipal de Saúde de Campinas.

Trata-se de um estudo observacional exploratório para análise de cobertura e resolubilidade dos encaminhamentos para a atenção secundária em Campinas (SP).

O município de Campinas possui área de $795,70 \mathrm{Km}^{2}$ e população de aproximadamente 1 milhão de habitantes ${ }^{12}$, distribuída em 5 Regiões Administrativas de Saúde (RAS): Norte, Sul, Leste, Sudoeste e Noroeste.

As RAS totalizam 61 Centros de Saúde (CS), 129 Equipes do Paidéia (Programa de atenção primária à saúde do município) e 61 Equipes de Saúde Bucal (ESB) ${ }^{13}$.

Estão estruturados para toda a rede os seguintes serviços especializados em saúde bucal: 5 referências de endodontia, 2 referências de periodontia, 5 referências de prótese e 2 Centros de Especialidades Odontológicas (CEO). O Hospital Municipal Dr. Mário Gatti é referência na área de cirurgia bucomaxilofacial para toda a rede, assim como o Serviço de diagnóstico bucal ACDC $^{14}$, conforme Tabela 1. 
Tabela 1. Oferta de Serviços especializados em saúde bucal disponibilizada no município de Campinas, pelas RAS Sudoeste e Norte no período de Julho de 2008 a junho de 2010, segundo seus respectivos Centros de Saúde.

\begin{tabular}{|c|c|c|c|c|c|c|c|c|c|c|}
\hline \multirow{2}{*}{ Unidade } & \multicolumn{10}{|c|}{ Especialidades Sudoeste } \\
\hline & Cirurgia & $\%$ & Endodontia & $\%$ & Prótese & $\%$ & Periodontia & $\%$ & Total & $\%$ \\
\hline Aeroporto & 5 & 0,75 & 141 & 13,58 & 34 & 9,55 & 32 & 3,87 & 212 & 7,34 \\
\hline Caic Vila União & 0 & 0,00 & 43 & 4,14 & 0 & 0,00 & 6 & 0,73 & 49 & 1,70 \\
\hline Capivari & 53 & 7,95 & 82 & 7,90 & 33 & 9,27 & 98 & 11,85 & 266 & 9,21 \\
\hline Dic I & 109 & 16,34 & 79 & 7,61 & 9 & 2,53 & 27 & 3,26 & 224 & 7,76 \\
\hline Dic III & 1 & 0,15 & 12 & 1,16 & 40 & 11,24 & 0 & 0,00 & 53 & 1,84 \\
\hline Itatinga & 11 & 1,65 & 68 & 6,55 & 33 & 9,27 & 37 & 4,47 & 149 & 5,16 \\
\hline Santa Lúcia & 98 & 14,69 & 135 & 13,01 & 50 & 14,04 & 136 & 16,44 & 419 & 14,51 \\
\hline Santo Antônio & 0 & 0,00 & 0 & 0,00 & 40 & 11,24 & 0 & 0,00 & 40 & 1,39 \\
\hline São Cristóvão & 12 & 1,80 & 47 & 4,53 & 2 & 0,56 & 38 & 4,59 & 99 & 3,43 \\
\hline Tancredo Neves & 75 & 11,24 & 77 & 7,42 & 22 & 6,18 & 46 & 5,56 & 220 & 7,62 \\
\hline Uniao de Bairros & 41 & 6,15 & 100 & 9,63 & 19 & 5,34 & 146 & 17,65 & 306 & 10,60 \\
\hline Vista Alegre & 61 & 9,15 & 80 & 7,71 & 36 & 10,11 & 98 & 11,85 & 275 & 9,52 \\
\hline Outros & 46 & 6,90 & 30 & 2,89 & 38 & 10,67 & 2 & 0,24 & 116 & 4,02 \\
\hline Total geral & 667 & 100,00 & 1038 & 100,00 & 356 & 100,00 & 827 & 100,00 & 2888 & 100,00 \\
\hline Faltas (\% geral) & & & & & $23,6 \%$ & & & & & \\
\hline \multirow{2}{*}{ Unidade } & \multicolumn{9}{|c|}{ Especialidades Norte } & \\
\hline & & Cirurgia & $\%$ & indodontia & $\%$ & Prótese & $\%$ & Total & $\%$ & \\
\hline Anchieta & & 87 & 24,30 & 178 & 11,90 & 9 & 6,52 & 274 & 13,74 & \\
\hline Aurélia & & 22 & 6,15 & 113 & 7,55 & 10 & 7,25 & 145 & 7,27 & \\
\hline Barão Geraldo & & 9 & 2,51 & 170 & 11,36 & 12 & 8,70 & 191 & 9,58 & \\
\hline Boa vista & & 28 & 7,82 & 82 & 5,48 & 33 & 23,91 & 143 & 7,17 & \\
\hline Cássio raposo & & 4 & 1,12 & 89 & 5,95 & 9 & 6,52 & 102 & 5,12 & \\
\hline Ceasa & & 9 & 2,51 & 58 & 3,88 & 4 & 2,90 & 71 & 3,56 & \\
\hline Eulina & & 23 & 6,42 & 120 & 8,02 & 16 & 11,59 & 161 & 8,07 & \\
\hline Santa Bárbara & & 50 & 13,97 & 165 & 11,03 & 15 & 10,87 & 230 & 11,53 & \\
\hline Santa Mônica & & 54 & 15,08 & 230 & 15,37 & 12 & 8,70 & 296 & 14,84 & \\
\hline São Marcos & & 71 & 19,83 & 275 & 18,38 & 18 & 13,04 & 364 & 18,25 & \\
\hline Village & & 1 & 0,28 & 16 & 1,07 & 0 & 0,00 & 17 & 0,85 & \\
\hline $\begin{array}{l}\text { Total geral } \\
\text { Faltas (\% geral) }\end{array}$ & & 358 & 100,00 & 1496 & $\begin{array}{r}100,00 \\
11,9 \%\end{array}$ & 138 & 100,00 & 1994 & 100,00 & \\
\hline
\end{tabular}

Foram selecionadas 02 RAS de Campinas, bem como todos os CS a elas pertencentes que apresentaram, no mínimo, 3 anos de atuação (tempo mínimo para análise de cobertura e resolutividade). Selecionou-se a RAS Sudoeste como a que possui CEO com mais de 3 anos de atuação e com 12 CS e em seguida, aleatoriamente uma RAS sem CEO implantado (Norte) com 11 CS.

Utilizou-se o período de encaminhamento de pacientes para as unidades de atenção secundária entre junho de 2008 a julho de 2010. Inicialmente, para analisar demanda atendida na atenção secundária (AS) em saúde bucal, foram identificados todos os sujeitos, sem restrição de gê- nero nem de idade, que procuraram os Centros de Saúde pertencentes às RAS selecionadas, que foram atendidos pelos Dentistas das Equipes de Saúde Bucal (ESB) e foram referenciados para tratamento de atenção secundária nas especialidades de endodontia, prótese, periodontia e cirurgia.

Essas referências estavam discriminadas no Sistema SOL - Sistema online de agendamento disponibilizado aos CS para agendamento nas especialidades odontológicas na região Sudoeste. Através deste sistema, as vagas para procedimentos especializados ficam disponíveis um dia na semana e sua distribuição por "cotas" para cada CS, sendo que as unidades básicas ficam 
responsáveis em entrar no sistema e agendar seus pacientes. As vagas não preenchidas entram novamente no sistema e são distribuídas por ordem de acesso ao sistema. Na Região Norte, as referências de endodontia e prótese também são geradas no sistema SOL, contudo, são retiradas do sistema e redistribuídas manualmente pelo gerente de saúde.

Os procedimentos das especialidades de Diagnóstico Oral e Radiologia não foram considerados, por serem caracterizados como apoio diagnóstico e terapêutico.

A lista de usuários contida no sistema foi fornecida pela Secretaria de Saúde de Campinas, onde constavam: nome, data do procedimento realizado, telefone de contato, especialidade e número do ticket (o qual permitiu identificar o CS de origem) e o número da Ficha Individual (FI) ou número da Ficha Familiar (FF) do paciente.

Em seguida foram coletadas informações sobre população adscrita a cada CS, dados socioeconômicos, cálculo de coeficientes de demanda atendida e percentual de faltosos, a fim de se analisar a distribuição da oferta de procedimentos pelos CS e RAS.

Foram calculados para cada CS 02 coeficientes, a saber: coeficiente total de procedimentos (procedimentos especializados totais por 100 habitantes), e coeficiente de endodontia (procedimentos de endodontia por 100 habitantes), pelo fato de ser a responsável pela maior parte dos procedimentos especializados realizados.

O município de Campinas utiliza o Índice de Condição de Vida (ICV) como método para identificação de diferenciais nos níveis de qualidade de vida e saúde das áreas de abrangência dos seus Centros de Saúde. O mesmo é composto por 8 indicadores selecionados dentre um conjunto de dados facilmente disponíveis nos sistemas de informações existentes na Secretaria $\mathrm{Mu}$ nicipal de Saúde (SMS): proporção de população moradora em sub-habitação; proporção de chefes de família sem ou com menos de 1 ano de instrução; taxa de crescimento anual entre os anos de 1991- 1996 ; proporção média de mães com menos de 20 anos de idade; coeficiente médio de mortalidade infantil; coeficiente médio de mortalidade por homicídios; incidência média de desnutrição entre os menores de 5 anos; incidência média de tuberculose ${ }^{15}$.

Além desse índice, o trabalho também utilizou outros indicadores disponíveis no DATASUS: percentual de responsáveis com renda de até 02 salários mínimos, média de número de anos estudados, \% pessoa não alfabetizadas maiores de
5 anos, \% mulheres responsáveis pelo lar, \% casas com esgoto de rede geral, \% domicílios sem banheiro.

Para avaliar as informações sobre a qualidade da contrarreferência foi calculado um número amostral de 331 usuários (186 RAS Sudoeste e 145 RAS Norte), considerando-se uma resposta para a população geral com erro amostral de $5,5 \%$, tomando como critério de cálculo a proporção de 0,50 (50\% de mesma resposta a qualquer das perguntas) e nível de confiança de $95 \%$.

$\mathrm{Na}$ entrevista por meio de chamadas telefônicas, a pesquisadora se identificou, explicou o objetivo da entrevista, pediu autorização para a utilização das informações assegurando o sigilo absoluto e, em seguida, aplicou as seguintes perguntas:

1 -Você realizou o tratamento especializado?

2-Se realizou o tratamento, como foi seu atendimento?

3-Se realizou o tratamento, quanto teve que esperar pela $1^{\text {a }}$ consulta?

4-Se não realizou o tratamento, qual foi o motivo?

5- Para os pacientes que já haviam concluído o tratamento:

a- Você voltou a ver seu dentista após o tratamento especializado?

b- Se não procurou o dentista, por quê?

Para a avaliação dos dados obtidos foi realizada a análise de correlação de Pearson entre os coeficientes de demanda atendida (coeficiente de endodontia e coeficiente total de referências especializadas) e as variáveis socioeconômicas da população de cada CS das RAS Sudoeste e Norte.

Para tanto, inicialmente, procedeu-se ao teste de multicolinearidade pela metodologia VIF (variance inflation factor), para as variáveis independentes, já que, dentre elas há variáveis que descrevem eventos similares. A análise do VIF apontou multicolinearidade aceitável entre as variáveis dependentes.

A seguir foi aplicada regressão linear múltipla pelo método Stepwise de seleção das variáveis. $\mathrm{O}$ ajuste do modelo foi avaliado pelo $\mathrm{C}_{\mathrm{p}}$ de Mellow e coeficiente de determinação $\left(\mathrm{R}^{2}\right)$.

\section{Resultados}

Podemos verificar na Tabela 1 que a especialidade Endodontia apresentou 35,9\% do total de procedimentos da região Sudoeste e 75,0\% da Norte, tendo sido a mais ofertada nas duas RAS.

As especialidades de cirurgia, prótese e periodontia foram responsáveis, respectivamente, por 
$23,0 \%, 12,3 \%$ e $28,6 \%$ na região Sudoeste. $\mathrm{Na}$ região Norte, as especialidades de cirurgia e prótese foram responsáveis por $17,9 \%$ e $6,9 \%$, respectivamente.

Em relação à distribuição das referências verifica-se que, na região Sudoeste, há uma discrepância na distribuição pelos diferentes CS (1,39 a $14,51 \%)$, o mesmo fato ocorrendo na região Norte $(7,17$ a $18,25 \%)$, levando em conta que nessa região duas CS (Village e Cássio Raposo) têm características próprias. Em ambas as RAS há clara discrepância também na distribuição pelas diferentes especialidades, onde para Cirurgia verifica-se variação na RAS Sudoeste de 0 a 16,34\%, enquanto que na RAS Norte variou de 2,51 a 24,3 $\%$. Para a especialidade Endontia variou de 0 a $13,58 \%$ na RAS Sudoeste e de 3,88 a 15,37\% na RAS Norte e, finalmente, para a especialidade Prótese variou de 0 a $14,04 \%$ para a RAS Sudoeste e de 2,90 a 23,91 \% para a RAS Norte.

$\mathrm{Na}$ Tabela 2, observa-se que, na região Sudoeste, a Unidade Itatinga ofertou cerca de 5 procedimentos especializados para cada 100 habitantes, enquanto Dic I, Dic III, São Cristovão e Santo Antonio ofertaram menos de 01 procedimento para cada 100 habitantes. Esse mesmo fato ocorreu na RAS Norte onde na CS ofertou-se cerca de 3,7 proc/100 hab, enquanto que nas CS Aurélia, Barão Geraldo, Eulina e Santa Bárbara ofertaram menos de 1 proc/100 hab. Em relação ao coeficiente de endodontia, na região Norte havia variação de 0,29 a 2,88 endodontias para cada 100 habitantes (Aurélia e Santa Mônica, respectivamente), e na Sudoeste, variação de 0 a 2,27 procedimentos (Santo Antonio e Itatinga).

Após verificadas as correlações de Pearson, a análise de regressão múltipla demonstrou que o coeficiente de endodontia apresentou $\mathrm{R}^{2} \mathrm{de}$ $94,88 \%$ e o coeficiente de Procedimentos Referenciados Totais apresentou $\mathrm{R}^{2}$ de $94,02 \%$, sendo que demonstrou-se que, na região Norte, há correlação estatisticamente significante entre o coeficiente de endodontia e coeficiente total de procedimentos com as variáveis "média de anos de estudo" e "percentual de responsáveis com renda de até 2 salários mínimos" (Tabela 3). Já na região Sudoeste, não foi encontrada correlação estatisticamente significativa entre nenhuma das variáveis estudadas.

Em relação à qualidade da contrarreferência, na Tabela 4 verifica-se que $78,62 \%$ e $51,61 \%$ dos entrevistados realizaram o tratamento especializado agendado nas regiões Norte e Sudoeste, respectivamente (Questão 1). Todos os entrevistados consideraram-no "muito bom" (Questão 2).
Quanto ao tempo de espera, na região Norte o mesmo foi menor $(p<0,005)$, sendo que $73,98 \%$ dos usuários dessa RAS reportaram esperar até um mês pelo agendamento, enquanto que para os da região Sudoeste este tempo de espera se deu para 52,00\% dos usuários. O maior tempo de espera pesquisado - de 45 dias - foi o relatado por $47 \%$ dos usuários da Sudoeste e por apenas $17,07 \%$ dos usuários da Norte (Questão 3).

O absenteísmo nas RAS Norte e Sudoeste foi de $11,9 \%$ e $23,6 \%$, respectivamente. Houve justificativa para as mesmas em $67,74 \%$ dos casos na RAS Norte e em $31,11 \%$ dos casos na RAS Sudoeste. Contudo, na RAS Sudoeste 29 relataram que não foram avisados e 10 não estavam esperando a vaga. Tal contingente representou $43,33 \%$ do total de usuários que não realizaram o tratamento (Questão 4).

Dos usuários que realizaram tratamento, 181 voltaram para a ESB de origem, sendo que $87,71 \%$ destes eram da RAS Norte e $84,37 \%$ da RAS Sudoeste (Questão 5a). Daqueles que não retornaram para a Unidade de origem, 07 relataram falta de tempo, 08 procuraram tratamento particular, 03 não voltaram em razão de compromissos com o trabalho e 04 por problemas particulares; 03 não receberam contrarreferência quando da finalização do tratamento especializado; 02 não voltaram por desinteresse e 02 devido à demora na marcação da consulta (Questão 5b).

\section{Discussão}

Este estudo teve como principal característica a avaliação objetiva dos indicadores de atenção secundária em saúde bucal em Campinas, buscando subsídio nos indicadores quantitativos absolutos ( $1^{\mathrm{a}}$ avaliação), relativos ( $2^{\mathrm{a}}$ avaliação) e relação da oferta com variáveis sociais ( $3^{\text {a }}$ avaliação); além de dados referentes à qualidade da contrarreferência ( $4^{\mathrm{a}}$ avaliação). Esta abordagem é original e necessária, no sentido de se contemplar avaliações de acesso e processo de trabalho em diferentes níveis de complexidade.

Considerando-se que a relação entre a atenção básica e os demais níveis da atenção não deve se restringir a fluxos e sim a processos; e que estes devem ser dinâmicos, democráticos e fundados na equidade, entende-se que o que deve haver entre os níveis de atenção não é superposição, mas interface, e que esta deve configurar-se como o lugar privilegiado para a ocorrência das mudanças necessárias à implementação da aten- 
Tabela 2. Informações populacionais, socioeconômicas e demográficas das Unidades de Saúde da Família das Regiões Norte e Sudoeste, Campinas, SP.

\begin{tabular}{|c|c|c|c|c|c|c|}
\hline Unidade Básica & \multicolumn{2}{|c|}{ Distrito } & $\begin{array}{c}\text { População estimada } \\
2010 \text { (IBGE) }\end{array}$ & \multicolumn{2}{|c|}{ Endodontias } & $\begin{array}{c}\text { coeficiente } \\
n^{\circ} \text { endo/100 hab }\end{array}$ \\
\hline CS C Raposo Amaral ${ }^{\#}$ & \multirow{9}{*}{\multicolumn{2}{|c|}{ Norte }} & $4.799^{\#}$ & \multicolumn{2}{|c|}{$89^{\#}$} & $1,85^{\#}$ \\
\hline CS S Marcos & & & 12.378 & \multicolumn{2}{|c|}{275} & 2,22 \\
\hline CS S Monica & & & 9.984 & \multicolumn{2}{|c|}{288} & 2,88 \\
\hline CS B Vista & & & 10.345 & \multicolumn{2}{|c|}{82} & 0,79 \\
\hline CS S Barbara & & & 24.644 & \multicolumn{2}{|c|}{165} & 0,67 \\
\hline CS Eulina & & & 19.294 & \multicolumn{2}{|c|}{120} & 0,62 \\
\hline CS Anchieta & & & 20.618 & \multicolumn{2}{|c|}{178} & 0,86 \\
\hline CS B Geraldo & & & 34.483 & \multicolumn{2}{|c|}{170} & 0,49 \\
\hline CS Aurelia & & & 38.765 & \multicolumn{2}{|c|}{113} & 0,29 \\
\hline CS Itatinga & \multicolumn{2}{|c|}{ Sudoeste } & 2.992 & \multicolumn{2}{|c|}{68} & 2,27 \\
\hline CS Capivari & \multicolumn{2}{|c|}{ suaveste } & 13.588 & \multicolumn{2}{|c|}{82} & 0,60 \\
\hline CS S Antonio & & & $10.743^{\#}$ & \multicolumn{2}{|c|}{$0^{\#}$} & $0,00^{\#}$ \\
\hline CS T Neves & & & 21.745 & \multicolumn{2}{|c|}{77} & 0,35 \\
\hline CS Aeroporto & & & 15.380 & \multicolumn{2}{|c|}{141} & 0,92 \\
\hline CS V Alegre & & & 20.247 & \multicolumn{2}{|c|}{80} & 0,40 \\
\hline CS S Cristovao & & & 19.986 & \multicolumn{2}{|c|}{47} & 0,24 \\
\hline CS DIC I & & & 31.305 & \multicolumn{2}{|c|}{79} & 0,25 \\
\hline CS U Bairros & & & 28.009 & \multicolumn{2}{|c|}{100} & 0,36 \\
\hline CS V Uniao/CAIC & & & 13.462 & \multicolumn{2}{|c|}{43} & 0,32 \\
\hline CS S Lucia & & & 21.084 & & 5 & 0,64 \\
\hline CS DIC III & & & 22455 & & 2 & 0,05 \\
\hline Unidade Básica & Distrito & $\begin{array}{l}\text { No Proc } \\
\text { Espec }^{*}\end{array}$ & $\begin{array}{l}\text { coeficiente no } \\
\text { proc/ } 100 \text { hab }\end{array}$ & ICV & renda & escolaridade 1 \\
\hline CS C Raposo Amaral ${ }^{\#}$ & Norte & $102^{\#}$ & $2,13^{\#}$ & $40^{\#}$ & não há dados ${ }^{\#}$ & não há dados ${ }^{\#}$ \\
\hline CS S Marcos & & 364 & 2,94 & 40 & 34,8 & 4,9 \\
\hline CS S Monica & & 367 & 3,68 & 40 & 35,9 & 4,9 \\
\hline CS B Vista & & 143 & 1,38 & 15 & 20,3 & 6,8 \\
\hline CS S Barbara & & 230 & 0,93 & 30 & 22,1 & 6,1 \\
\hline CS Eulina & & 165 & 0,86 & 7,5 & 11,1 & 9,6 \\
\hline CS Anchieta & & 274 & 1,33 & 40 & 25,4 & 5,9 \\
\hline CS B Geraldo & & 191 & 0,55 & 7,5 & 11,3 & 10,1 \\
\hline CS Aurelia & & 145 & 0,37 & 7,5 & 11,6 & 9,4 \\
\hline CS Itatinga & Sudoeste & 149 & 4,98 & 40 & 38,4 & 4,7 \\
\hline CS Capivari & & 266 & 1,96 & 30 & 22,8 & 5,8 \\
\hline CS S Antonio ${ }^{\#}$ & & $40^{\#}$ & $0,37^{\#}$ & $40^{\#}$ & não há dados” & \\
\hline CS T Neves & & 220 & 1,01 & 15 & 13,8 & 7,8 \\
\hline CS Aeroporto & & 212 & 1,38 & 40 & 24,1 & 5,7 \\
\hline CS V Alegre & & 275 & 1,36 & 40 & 25,3 & 5,7 \\
\hline CS S Cristovao & & 99 & 0,50 & 40 & 27,1 & 5,1 \\
\hline CS DIC I & & 224 & 0,72 & 40 & 23,6 & 6,1 \\
\hline CS U Bairros & & 306 & 1,09 & 40 & 36,6 & 5 \\
\hline CS V Uniao/CAIC & & 49 & 0,36 & 15 & 8,1 & 8,8 \\
\hline CS S Lucia & & 419 & 1,99 & 30 & 24,1 & 5,7 \\
\hline CS DIC III & & 53 & 0,24 & 40 & 22,6 & 5,9 \\
\hline
\end{tabular}

continua

ção secundária em saúde bucal. Ao pesquisar esse "lugar" onde a relação entre os níveis ocorre, o presente trabalho identificou, no município de Campinas, características importantes relaciona- das a processo de trabalho, referência e contrarreferência, organização da demanda e outros, cuja elaboração poderá trazer soluções a importantes entraves à integralidade. 
Tabela 2. Continuação

\begin{tabular}{|c|c|c|c|c|c|c|}
\hline Unidade Básica & Distrito & $\begin{array}{c}\text { escolaridade } \\
2\end{array}$ & $\begin{array}{c}\text { demográfico } \\
1\end{array}$ & $\begin{array}{c}\text { demográfico } \\
2\end{array}$ & $\begin{array}{c}\text { demográfico } \\
3\end{array}$ & $\begin{array}{c}\% \text { pop } \\
>65 \text { anos }\end{array}$ \\
\hline CS C Raposo Amaral ${ }^{\#}$ & Norte & não há dados & não há dados" & não há dados ${ }^{\#}$ & não há dados ${ }^{\#}$ & não há dados" \\
\hline CS S Marcos & & 26,1 & 22,7 & 78,2 & 3,8 & 4,9 \\
\hline CS S Monica & & 6,9 & 24,4 & 65,6 & 1,6 & 8,1 \\
\hline CS B Vista & & 5,9 & 24,7 & 95,3 & 1,5 & 8,1 \\
\hline CS S Barbara & & 8 & 17,6 & 76,2 & 2,9 & 2,9 \\
\hline CS Eulina & & 0,6 & 21,7 & 98 & 0,8 & 9,6 \\
\hline CS Anchieta & & 5,6 & 26,2 & 86,9 & 1,3 & 5 \\
\hline CS B Geraldo & & 11 & 19,9 & 67,1 & 0,8 & 5,6 \\
\hline CS Aurelia & & 4,8 & 28,4 & 99,7 & 0,5 & 10,9 \\
\hline CS Itatinga & Sudoeste & 13,2 & 25,8 & 96,8 & 1,3 & 2,9 \\
\hline CS Capivari & & 11,1 & 23,9 & 94,6 & 1,1 & 5,2 \\
\hline CS S Antonio" & & não há dados" & não há dados ${ }^{\#}$ & não há dados ${ }^{\#}$ & não há dados ${ }^{\#}$ & não há dados ${ }^{\#}$ \\
\hline CS T Neves & & 6,4 & 21,4 & 97,3 & 0,4 & 5,9 \\
\hline CS Aeroporto & & 22 & 19 & 92,4 & 1,1 & 3,7 \\
\hline CS V Alegre & & 11,4 & 19,6 & 89,4 & 1,7 & 3,5 \\
\hline CS S Cristovao & & 8 & 16,1 & 83,8 & 0,8 & 2,8 \\
\hline CS DIC I & & 19,6 & 24,2 & 86,3 & 2,2 & 3,1 \\
\hline CS U Bairros & & 20,9 & 19,9 & 60,2 & 2,1 & 2 \\
\hline CS V Uniao/CAIC & & 9,5 & 23,8 & 99,7 & 0,3 & 2,1 \\
\hline CS S Lucia & & 15 & 20,8 & 94,8 & 0,9 & 4,4 \\
\hline CS DIC III & & 4,6 & 21,7 & 72,6 & 1,6 & 2,5 \\
\hline
\end{tabular}

Renda: \% de responsáveis com renda até 2SM; Escolaridade 1: média de número de anos estudados; Escolaridade 2: \% Pessoa não alfabetizadas maiores de 5 anos; Demográfico 1: \% mulheres responsáveis pelo lar; Demográfico 2: \% casas com esgoto de rede geral; Demográfico 3: \% domicílios sem banheiro. ICV: Índice de Condição de Vida. * corresponde a Unidades inauguradas após o ano 2000 (ano em que o sistema apresenta dados socioeconômicos e demográficos). Variáveis socioeconômicas (extraídas através pelo Tabnet através do site da Prefeitura Municipal de Saúde de Campinas - dado do ano 2000)

Tabela 3. Parâmetros estimados pela análise de regressão múltipla método stepwise para os coeficientes de endodontia e de procedimentos referenciados totais na região Norte.

\begin{tabular}{lrrr}
\hline & Coeficiente & Erro padrão & p-valor \\
\hline Coeficiente de endodontia $\left(\mathrm{R}^{2}=0,9488\right)$ & & & \\
& & & \\
Intercepto & $-1,4340$ & 0,5129 & 0,0129 \\
\% responsáveis com renda até 2SM & 0,0678 & 0,0149 & 0,0003 \\
média de número de anos estudados & 0,1361 & 0,0523 & 0,0192 \\
Coeficiente de procedimentos referenciados totais $\left(\mathrm{R}^{2}=0,9402\right)$ & & & \\
Intercepto & $-6,9202$ & 2,3224 & 0,0308 \\
\% responsáveis com renda até 2SM & 0,2155 & 0,0425 & 0,0039 \\
média de número de anos estudados & 0,5238 & 0,1982 & 0,0458 \\
\hline
\end{tabular}

Um dos grandes problemas quando se avalia a produção relativa a um serviço é o de se pautar toda a análise na produção por procedimento, sem levar em conta aspectos como porte populacional e fatores socioeconômicos da região adscrita. É importante analisar a oferta adequada de procedimentos segundo as necessidades populacionais, a acessibilidade geográfica e organizacio- nal ${ }^{16}$. Dessa forma, as Tabelas 2 e 3 demonstram claramente que a região Sudoeste apresentou grande variação na distribuição por CS e nos coeficientes de procedimentos especializados totais e endodontia por grupo de habitantes e que na região Norte essas diferenças não foram tão acentuadas, embora tenha sido verificado um desequilíbrio na distribuição. 
Tabela 4. Respostas dos usuários para perguntas acêrca da qualidade da contrarreferência em atenção secundária no município de Campinas, segundo as regiões administrativas de saúde Norte e Sudoeste.

\begin{tabular}{lccc}
\hline \multicolumn{4}{c}{ Q1- Você realizou tratamento especializado? } \\
\hline \multicolumn{1}{c}{ Região } & Sim N (\%) & Não N (\%) & Total \\
\hline Norte & $114(78,62)$ & $31(21,37)$ & 145 \\
Sudoeste & $96(51,61)$ & $90(48,38)$ & 186 \\
Total & $210(63,44)$ & $121(36,56)$ & 331 \\
\hline
\end{tabular}

Q3- Se realizou o tratamento, quanto tempo esperou para que a consulta fosse marcada?

\begin{tabular}{lcccc}
\hline & Até um mês $\mathbf{N}(\%)$ & Até $\mathbf{4 5}$ dias $\mathbf{N}(\%)$ & Mais de $\mathbf{4 5}$ dias $\mathbf{N}(\%)$ & Total \\
\hline Norte & $91(73,98)$ & $11(8,94)$ & $21(17,07)$ & 123 \\
Sudoeste & $52(52,00)$ & $1(1,00)$ & $47(47,00)$ & 100 \\
Total & $143(64,12)$ & $12(5,38)$ & $68(30,49)$ & 223 \\
\hline
\end{tabular}

Q4- Se você não realizou o tratamento, qual o motivo?

\begin{tabular}{|c|c|c|c|c|}
\hline & Faltou N (\%) & Indicação errada N (\%) & Longe $N(\%)$ & Foi no particular $\mathrm{N}(\%)$ \\
\hline Norte & $21(67,74)$ & $4(12,9)$ & $1(3,22)$ & $2(6,55)$ \\
\hline \multirow[t]{2}{*}{ Sudoeste } & $28(31,11)$ & $4(4,44)$ & 0 & $9(10,00)$ \\
\hline & $\begin{array}{c}\text { Demorou } \\
\text { para marcar }\end{array}$ & $\begin{array}{l}\text { Não quis fazer } \\
\text { o tratamento }\end{array}$ & $\begin{array}{l}\text { Não foi } \\
\text { avisado }\end{array}$ & $\begin{array}{c}\text { Não esperava } \\
\text { vaga }\end{array}$ \\
\hline Norte & $1(3,22)$ & $2(6,45)$ & 0 & 0 \\
\hline Sudoeste & $10(11,11)$ & 0 & $29(32,22)$ & $10(11,11)$ \\
\hline
\end{tabular}

\begin{tabular}{|c|c|c|c|c|c|}
\hline \multicolumn{6}{|c|}{ Q5 a - Você voltou a ver o seu CD após o tratamento especializado? } \\
\hline & \multicolumn{2}{|l|}{ Sim N $(\%)$} & \multicolumn{2}{|l|}{ Não N (\%) } & Total \\
\hline Norte & \multicolumn{2}{|l|}{$100(87,71)$} & \multicolumn{2}{|l|}{$14(12,28)$} & $114(100)$ \\
\hline Sudoeste & \multicolumn{2}{|l|}{$81(84,37)$} & $15(15,62)$ & \multicolumn{2}{|r|}{$96(100)$} \\
\hline \multicolumn{6}{|c|}{ Q 5b - Se não procurou o CD, por quê? } \\
\hline & $\begin{array}{c}\text { Falta de tempo } \\
\text { N (\%) }\end{array}$ & $\begin{array}{l}\text { Problemas particulares } \\
\text { N }(\%)\end{array}$ & $\begin{array}{l}\text { Trabalho } \\
\mathrm{N}(\%)\end{array}$ & \multicolumn{2}{|c|}{$\begin{array}{c}\text { Procurou CD particular } \\
\text { N (\%) }\end{array}$} \\
\hline Norte & $4(28,57)$ & $2(14,28)$ & $2(14,28)$ & $2(14$, & 28) \\
\hline \multirow[t]{2}{*}{ Sudoeste } & $3(20,00)$ & $2(13,33)$ & $1(6,66)$ & $6(40$ & \\
\hline & $\begin{array}{c}\text { Demorou para marcar } \\
\qquad \mathrm{N}(\%)\end{array}$ & $\begin{array}{l}\text { Desinteresse } \\
\text { N (\%) }\end{array}$ & \multicolumn{2}{|c|}{$\begin{array}{l}\text { Paciente sem contra-referência } \\
\qquad N(\%)\end{array}$} & $\begin{array}{l}\text { Total } \\
\text { N }(\%)\end{array}$ \\
\hline Norte & 0 & $2(14,28)$ & \multicolumn{2}{|c|}{$2(14,28)$} & $14(100)$ \\
\hline Sudoeste & $2(13,33)$ & 0 & \multicolumn{2}{|c|}{$1(6,66)$} & $15(100)$ \\
\hline
\end{tabular}

Outro aspecto importante a se levar em consideração é a equidade na organização da demanda, e para isso a observação das variáveis socioeconômicas é de grande relevância para a análise do acesso na distribuição dos procedimentos especializados. Sendo assim, as variáveis "ICV”, "percentual de responsáveis com renda até 2 Salários Mínimos" e "média em número de anos estudados do responsável” foram estatisticamente correlacionadas com a distribuição dos procedimen- tos endodônticos e na distribuição total das referências na região Norte, enquanto nenhuma correlação foi verificada na região Sudoeste. Isso foi ainda mais claro quando se observou que as duas últimas variáveis foram confirmadas no modelo de regressão linear múltipla. Isto demonstra maior preocupação com o princípio da equidade na Região Norte, onde as áreas menos favorecidas receberam um número maior de referências especializadas com melhor distribuição. 
Nas duas regiões pesquisadas, todos os atendimentos realizados nas especialidades foram referenciados, não havendo, portanto, demanda espontânea para os mesmos. $\mathrm{Na}$ agenda da região Sudoeste, onde os agendamentos eram feitos pelos CS, observou-se muitos nomes repetidos para um mesmo procedimento, verificando-se que as unidades realizavam tal prática para garantir os agendamentos no sistema SOL, e depois redistribuírem para os pacientes. Essa prática dificultou sobremaneira a checagem da contrarreferência, já que impossibilitou a identificação de todos os usuários que receberam tratamento. Tais resultados induzem a reflexão sobre a necessidade de se adequar normas e critérios para implantação e monitoramento desses serviços e vem de encontro com estudo de Figueiredo e Goes ${ }^{17}$ sobre a função dos Centros de Especialidades Odontológicas como estratégia na oferta da atenção secundária em saúde bucal, os quais, inclusive, sugerem novas pesquisas quanto ao uso de protocolos clínicos específicos e efetivos de referência e contrarreferência.

Além disso, também ficou constatado que, para as vagas inicialmente não ocupadas, as unidades que entrassem primeiramente no sistema SOL seriam as mais beneficiadas, não priorizando o acesso a quem mais necessitava, já que dessa forma não se consideravam as variáveis socioeconômicas e a demanda reprimida. A não adequação da oferta das referências aos grupos mais necessitados parece ser comum no Brasil, conforme concluíra Costa e Facchini ${ }^{18}$, onde classes sociais mais baixas têm 25\% menos chance de serem consultadas por um médico.

Na região Norte de Campinas, onde as vagas são centralizadas no distrito e depois distribuídas, foi constatada correlação entre os procedimentos de cada CS e a variável socioeconômica, propiciando um melhor gerenciamento e mais equidade na oferta de referências para procedimentos especializados.

A organização da demanda da atenção secundária em saúde bucal foi diferente entre as RAS, sendo isso ainda melhor constatado quando se analisam as informações de referência e contrarreferência.

Nos CS da região Sudoeste não havia anotação alguma sobre os pacientes referenciados para as especialidades. Já nos CS da região Norte, parte dos pacientes referenciados - especialmente os de endodontia - eram registrados em arquivo próprio pelas Auxiliares de Saúde Bucal (ASB), constando de nome, data e procedimento solicitado. Entretanto, não era anotado o número da ficha individual (FI) ou familiar (FF) deste paciente. Isso demonstra um importante problema relacionado a processo de trabalho: a desorganização e a precariedade dos arquivos, cujas consequências vão desde a dificuldade de contato com o usuário para eventuais avisos, até a impossibilidade de planejamento da oferta, uma vez que a falta de informações compromete o reconhecimento real das necessidades. Assim, foi necessário solicitar ao apoiador distrital, dados para o contato telefônico e entrevista com os usuários, onde se verificou a resolutividade dos tratamentos referenciados, a qualidade do atendimento, o tempo de espera e as razões para faltas/desistência ou não retornos.

A maioria dos usuários referenciados realizou os tratamentos especializados agendados. Em relação aos pacientes que não realizaram o tratamento, a maioria foi por esquecimento ou outros fatores de ordem externa, como mau tempo ou compromisso de última hora. Esses dados estão de acordo com Almeida et al. ${ }^{19}$, em 2009, que apontaram que $59,1 \%$ das faltas dos pacientes em consultas odontológicas em Ribeirão Preto (SP), eram por esquecimento; $13,6 \%$ eram devidas a compromissos com o trabalho e $4,5 \%$ devidas a horário inadequado ou outros motivos.

Outras duas questões - menos óbvias, mas muito relevantes - foram apuradas na região Sudoeste: 29 pacientes relataram que não sabiam da consulta, pois não foram avisados; e outros 10 pacientes não estavam esperando a vaga para tratamento especializado e/ou nem mesmo tinham feito qualquer tratamento odontológico pelo SUS. Esse contingente correspondia a 43,3\% do total de pacientes que não realizou o tratamento. Esse ponto foi o mais crítico entre todos os dados encontrados, pois demonstrou o descumprimento de princípios básicos da gestão pública, especificamente a organização da demanda, não sendo aceitável que tantas vagas para agendamento das referências sejam perdidas, seja por falha na comunicação com os usuários, seja pela inclusão inadvertida de indivíduos nas referências ou, ainda, por "burla" do sistema, com inserção de nomes repetidos para reserva de vagas. Essas falhas de organização possivelmente culminaram no alto percentual de faltas (23,6\%); além da correlação da demanda atendida com a equidade. Portanto, sugere-se uma discussão crítica nessa RAS no sentido de uma readequação do modelo de gestão da atenção secundária.

O fato do estudo ter sido realizado em Campinas não limita sua inferência, pois a análise não se pauta em comparações locais, mas sim no pro- 
cesso de trabalho, o qual pode e deve ser inferido e reproduzido em qualquer outro município do país.

\section{Conclusão}

Conclui-se que o modelo de gestão, com ou sem a instalação do CEO, não influenciou na distribuição das referências da atenção secundária em saúde bucal quando se comparou as duas RAS da cidade de Campinas, mas que o processo de trabalho certamente influenciou. Houve claras inconsistências nas distribuições dos atendimentos de referência por CS, quando se observavam os valores absolutos e os coeficientes de atendimento especializado geral e de endodontia por grupo de 100 habitantes em ambas as RAS. Verificou-se que a Região Norte, onde há o gerenciamento das vagas, as discrepâncias são menos evidentes, havendo uma correlação dos coeficientes de demanda atendida em relação às variáveis socioeconômicas, demonstrando um maior compromisso com a integralidade e equidade. Ao analisar-se a qualidade da referência e contrarreferência, a grande maioria dos usuários das duas RAS realizou o tratamento especializado, retornou ao CD da ESB de origem, foi bem atendido; contudo na RAS Sudoeste algumas razões para o não comparecimento ao tratamento não foram convencionais (não esperavam atendimento, não foram avisados e outros) e deveriam ser analisados no contexto administrativo pelos gestores das RAS.

\section{Colaboradores}

FL Vazquez, LM Guerra, E Sant'Anna Vítor, GMB Ambrosano, FL Mialhe, MC Meneghim e AC Pereira participaram igualmente de todas as etapas de elaboração do artigo. 


\section{Referências}

1. Brasil. Ministério da Saúde (MS). Secretaria de Atenção à Saúde. Departamento de Atenção Básica. Coordenação Nacional de Saúde Bucal. Pesquisa Nacional de Saúde Bucal - 2010. Nota para a imprensa. Brasília: MS; 2010.

2. Pezzato LM, L’Abbate S, Botazzo C. Produção de micropolíticas no processo de trabalho em saúde bucal: uma abordagem socioanalítica. Cien Saude Colet 2013; 18(7):2095-2104.

3. Narvai PC. Odontologia e Saúde Coletiva. São Paulo: Hucitec; 1994.

4. Brasil. Ministério da Saúde (MS). Secretaria de Atenção à Saúde. Departamento de Atenção Básica. Saúde da Família. Números da Saúde da Família. [página na Internet]. [acessado 2012 out 11]. Disponível em: http://200.214.130.135/dab/abnumeros. php\#numeros

5. Junqueira SR, Frias AC, Zilbovicius C, Araujo ME. Saúde bucal e uso dos serviços odontológicos em função do Índice de Necessidades em Saúde: São Paulo, 2008. Cien Saude Colet 2012; 17(4):1015-1024.

6. Soares FF, Figueiredo CRV, Borges NCM, Jordão RA, Freire MCM. Atuação da equipe de saúde bucal na estratégia saúde da família: análise dos estudos publicados no período 2001-2008. Cien Saude Colet 2011; 16(7):3169-3180.

7. Brasil. Ministério da Saúde (MS). Secretaria de Atenção à Saúde. Departamento de Atenção Básica. Coordenação Nacional de Saúde Bucal. Diretrizes da Política Nacional de Saúde Bucal. Brasília: MS; 2004.

8. Souza TMS, Roncalli AG. Saúde bucal no Programa de Saúde da Família: uma avaliação do modelo assistencial. Cad Saude Publica 2007; 23(11):27272739.

9. Fratini JRG, Saupe R, Massaroli, A. Referência e Contrarreferência: Contribuição para a Integralidade em Saúde. Cienc Cuid Saude 2008; 7(1):65-72.

10. Juliani, CMCM, Ciampone MHT. Organização do sistema de referência e contrarreferência no contexto do Sistema Único de Saúde: a percepção de enfermeiros. Rev. Esc. Enf. 1999; 33(4):323-333.

11. Marra SMP, Arcieri RM. Análise da inclusão da odontologia nos planos municipais de saúde das cidades integrantes da gerência regional de saúde de Uberlândia-MG. Biosci J 2011; 27(3):510-517.

12. São Paulo (Estado). Secretaria de Economia e Planejamento. Fundação Sistema Estadual de Análise de Dados (SEADE). Perfil Municipal. Campinas. [página na Internet]. [acessado 2010 set 26]. Disponível em: http://www.seade.sp.gov.br/produtos/ perfil/perfil.php
13. Campinas. Secretaria Municipal de Saúde. SUS em Campinas. Unidades básicas de Saúde. [página na Internet]. [acessado 2010 set 26]. Disponível em: http://2009.campinas.sp.gov.br/saude/

14. Campinas. Secretaria Municipal de Saúde. SUS em Campinas. Estrutura do SUS. [página na Internet]. [acessado 2010 set 26]. Disponível em: http:// 2009.campinas.sp.gov.br/saude/

15. Campinas. Secretaria Municipal de Saúde. SUS em Campinas. Estrutura do SUS. [página na Internet]. [acessado 2012 nov 20]. Disponível em: http:// www.dadosmunicipais.org.br/index.php?pg= exibemateria\&secao $=10 \&$ subsecao $=\& i d=373 \&$ \&id $=$

16. Mendoza-Sassi R, Beria JU. Utilización de los servicios de salud: una revisión sistemática sobre los factores relacionados. Cad Saude Publica 2001; 17(4):819-832.

17. Figueiredo N, Goes PSA. Construção da atenção secundária em saúde bucal: um estudo sobre os Centros de Especialidades Odontológicas em Pernambuco, Brasil Faculdade de Odontologia, Universidade de Pernambuco, Camaragibe, Brasil. Cad Saude Publica 2009; 25(2):259-267.

18. Costa JD, Facchini LA. Utilização de serviços ambulatoriais em Pelotas: onde a população consulta e com que freqüência. Rev Saude Publica 1997; 31(4):360-369.

19. Almeida GL, Garcia LFR, Almeida TL, Bittar TO, Pereira AC. Estudo do perfil sócio-econômico dos pacientes e os motivos que os levaram a faltar em consultas odontológicas na estratégia de saúde da família em uma distrital de Ribeirão Preto/SP. Cienc Odontol Bras 2009; 12(1):77-86.

Artigo apresentado em 10/08/2012

Aprovado em 13/10/2012

Versão final apresentada em 29/10/2012 
